\section{A transnacionalização do parto normal no Brasil: um estudo das últimas cinco décadas}

\section{The transnationalization of normal childbirth in Brazil: a study of the last five decades}

Sara Fernandes Picheth

Doutoranda, Programa de Pós-graduação em Administração (PPA)/ Universidade Estadual de Maringá (UEM). Maringá - PR - Brasil

sarafpicheth@gmail.com

João Marcelo Crubellate

Professor, Departamento de Administração e PPA/UEM. Maringá - PR - Brasil

jmcrubellate@uem.br

\section{Fabiane Cortez Verdu}

Professora, Departamento de Administração e PPA/UEM. Maringá - PR - Brasil

fabiane.verdu@gmail.com

Recebido em 27 mar. 2017.

Aprovado em 5 jan. 2018.
PICHETH, Sara Fernandes; CRUBELLATE, João Marcelo; VERDU, Fabiane Cortez. A transnacionalização do parto normal no Brasil: um estudo das últimas cinco décadas. História, Ciências, Saúde Manguinhos, Rio de Janeiro, v.25, n.4, out.-dez. 2018, p.1063-1082.

Resumo

Nos últimos anos têm ocorrido mudanças no sistema obstétrico brasileiro, em função da distância entre o cenário nacional e recomendações da Organização Mundial da Saúde e da atuação de movimentos sociais. Isso evidencia a necessidade de considerar tanto o contexto nacional como o internacional. A fim de compreender a influência da transnacionalização no parto normal no Brasil, conduziu-se uma pesquisa documental para rastrear a evolução do sistema obstétrico no país. Como resultado, observou-se que a trajetória histórica do parto normal não se restringiu às fronteiras nacionais, tendo padrões e recomendações transnacionais impactado localmente, direcionando a criação de novas regulamentações.

Palavras-chave: transnacionalização; governança transnacional; parto normal; movimento pela humanização do parto e do nascimento.

\section{Abstract}

In recent years, changes have taken place in Brazilian obstetrics in response to the distance between the national scenario and World Health Organization recommendations and the work of organized civil society. This indicates the need for the national and international contexts to be considered. As such, the aim here is to understand the influence of transnationalism on normal childbirth in Brazil. Documental research was conducted to trace out the development of obstetrics in the country. It was found that the historical trajectory of normal childbirth in Brazil has not necessarily been restricted to national borders, but has been influenced by transnational recommendations and standards, orienting the creation of new regulations.

Keywords: transnationalization; transnational governance; normal childbirth; movement for the humanization of childbirth and birth. 
$\mathrm{A}$ preocupação com o alto índice de cesarianas realizadas no Brasil tem sido foco de crescente número de estudos. Retratadas na maioria das vezes como uma "epidemia", as cesáreas se tornaram, ao longo das últimas décadas, a principal via de nascimento no país (Frasão, 2016). Esses estudos concernem tanto ao nível acadêmico (Rattner, 1996; Sakae, Freitas, D’Orsi, 2009; Mendoza-Sassi et al., 2010; Barros et al., 2011) quanto à mídia popular e aos sites de órgãos nacionais (ANS publica..., s.d.; Frasão, 2016; Nascer..., 1976; Junqueira, 1997).

Se em 1970 o índice de cesarianas realizadas em hospitais públicos brasileiros era de aproximadamente 14,6\%, em 1980 ele saltou para 31\% (Faúndes, Cecatti, 1991). Essa tendência crescente perdura até o presente. Pesquisas revelam que, em 2009, esse índice aumentou para 50,1\% do total de partos, tornando o Brasil o primeiro país no mundo a ter mais da metade dos bebês nascendo de modo não natural. Números atuais apontam que o Brasil lidera o ranking mundial de cesáreas, com 52\% dos partos ocorrendo por via cirúrgica, sendo que em redes privadas a situação é ainda mais alarmante, chegando a 88\% (Leal, Gama, 2012).

Esses valores evidenciam que o Brasil se encontra bem distante do percentual recomendado pela Organização Mundial da Saúde (OMS), definido, desde 1985, entre 10 a 15\% (WHO, 2015). Mais recentemente, a partir de uma avaliação específica do país, a OMS ajustou essa taxa de referência para o Brasil de 25 a 30\% (Frasão, 2016; Betran et al., 2015), ainda inferior ao cenário atual. A delimitação desses valores baseia-se em diversos estudos que identificaram associação entre taxas mais elevadas de mortalidade materna e neonatal a altas taxas de cesariana (Faúndes, Cecatti, 1991; Villar et al., 2006).

Consequentemente, a alta incidência de cesáreas no país tem sido apontada como um problema de saúde pública (ANS publica..., s.d.), levando diversos órgãos e instituições Ministério da Saúde, Agência Nacional de Saúde Suplementar (ANS) e Conselho Federal de Medicina (CFM) - a decretarem nos últimos anos mudanças no sistema obstétrico brasileiro, como as resoluções n.368 e n.398 (Brasil, 6 jan. 2015; Brasil, 5 fev. 2016; Atualização..., 17 maio 2015). É notável também a atuação de diversos movimentos e grupos sociais incentivando e propagando maior incidência de parto natural, humanização do parto, empoderamento da mulher e medicina baseada em evidências (Diniz, 2005; Goer, 2004; Brasil, 2001; Jones, 2007; RNFS, 2002). Esses movimentos, além de atuarem em um âmbito microssocial, divulgando informações e conscientizando seus participantes, também têm forte atuação na sociedade e no país, por meio de campanhas, eventos, ações, petições e audiências solicitadas e realizadas junto ao Ministério Público Federal, debatendo questões como violência obstétrica, altas taxas de cesáreas e humanização do nascimento (RNFS, 2002; Tornquist, 2004; MPF, 2010).

Todas essas ações de órgãos públicos e privados e dos movimentos sociais têm como aspectos comuns a defesa e a justificativa de mudanças a partir da comparação do cenário brasileiro com o de outros países e com as recomendações da OMS - braço da Organização das Nações Unidas (ONU) -, e ainda com os resultados de pesquisas realizadas e publicadas internacionalmente. Isso evidencia que, para estudar e compreender como o sistema obstétrico, ${ }^{1}$ em especial o parto normal, ${ }^{2}$ foi mudando ao longo dos anos no Brasil, é necessário considerar tanto o contexto nacional como o internacional. A reflexão crítica 
dessas mudanças deve abranger os vários atores com seus respectivos históricos e contextos, pois os atores se engajam localmente, mas seus esforços de governança atravessam fronteiras (Dobusch, Mader, Quack, 2013).

Segundo Zajak (2013, p.23), “a governança através das fronteiras ou governança transnacional olha para a criação de regras, definição de normas e construção de instituições através da fronteira ... A vida social sempre cruzou, conectou ou transformou fronteiras e limites". ${ }^{3}$ Desse modo, processos sociais são transfronteiriços e, mesmo nacionalmente, são moldados transnacionalmente também. No mundo transnacional, há a proliferação de regras (Djelic, Sahlin-Andersson, 2006b; Quack, 2013), bem como o aumento da variedade de atores interagindo para moldar as regras transnacionais e implementá-las (Quack, 2013). A esfera transnacional transcende as bordas nacionais ao mesmo tempo que se enreda em instituições históricas e é moldada por atores enraizados em diversos contextos e, portanto, captura melhor a realidade estudada (Dobusch, Mader, Quack, 2013).

Nesse sentido, este artigo pretende compreender a influência da transnacionalização no parto normal no Brasil. Sugere-se que a pesquisa científica social amplie sua visão para compreender a regulação como um "complexo conjunto de atividades que fazem a ponte entre o global e o local e que ocorrem ao mesmo tempo dentro, entre e através das fronteiras nacionais" (Djelic, Sahlin-Andersson, 2006b, p.5). Dessa forma, apresentam-se quatro questionamentos que buscam avançar e contribuir com o corpo de pesquisas em transnacionalização: como contextos internacionais interagem com a regulação nacional e como impactam sobre ela? Como as regras e instituições evoluem além e através das fronteiras? Como a governança transnacional ajuda a explicar as normas e os regulamentos utilizados pelo Ministério da Saúde e pela ANS no que se refere ao parto? E, finalmente, como o contexto internacional influencia na criação e na atuação de movimentos sociais?

Para responder a essas questões, conduziu-se um estudo para rastrear a evolução do sistema obstétrico no Brasil, analisando-se artigos, livros, teses, reportagens e regulamentos das áreas da saúde e, de modo mais sistemático, analisando-se publicações das últimas cinco décadas em quatro revistas brasileiras. Este estudo ilustra como o parto normal no Brasil - em especial suas últimas regulamentações -, mesmo inserido em um contexto nacional, sofre influência de contextos internacionais, devendo, portanto, ser analisado para além das fronteiras.

\section{Transnacionalização}

A transnacionalização do mundo não consiste apenas de fluxos de bens, de capital ou de pessoas, e de fato está longe disso. Também não concerne apenas a uma dimensão discursiva, embora essa seja de grande importância. Numa base experiencial, a maioria das pessoas sente o impacto de um mundo transnacional em suas vidas cotidianas (Djelic, Sahlin-Andersson, 2006a, 2006b). Cada vez mais é perceptível que desenvolvimentos econômicos, sociais e políticos que ocorrem em um determinado local sofrem influências de desenvolvimentos de outros locais. Assim, torna-se necessário ampliar o olhar a fim de analisar as ações de diversos atores em diferentes origens e locais para além das fronteiras, em uma governança transnacional (Dobusch, Mader, Quack, 2013). O mundo visto a 
partir dessa ótica é um mundo onde as regras institucionais estão em transição e passam por processos de reordenação que impactam de modo significativo o cotidiano das pessoas (Djelic, Sahlin-Andersson, 2006a).

Uma lente transnacional concebe o mundo sem fronteiras e limites, buscando compreender em quais contextos sócio-históricos particulares ele se insere e como esses interagem. Não se considera o espaço sob análise como dado, mas busca-se conhecer a geografia e as questões relevantes em que se encontra imerso. A governança transfronteira ou transnacional olha para a elaboração de normas, para a definição de padrões e para a criação de instituições através das fronteiras. Não há uma predileção pelo global ou pelo local, mas a interação de um com o outro é privilegiada, bem como toda experiência social que há entre ambos, isto é, considera-se global, nacional, regional e local como transnacionalmente constituídos, construídos por meio de de interações contínuas e iterativas (Zajak, 2013).

A transnacionalização, desse modo, implica que não há um domínio de culturas nacionais, tampouco a separação dessas (Dobusch, Mader, Quack, 2013). Ao contrário, ela transcende as fronteiras nacionais e, ao mesmo tempo, enreda-se em instituições historicamente contingentes que são moldadas por atores enraizados em contextos diversos, local e nacionalmente (Dobusch, Mader, Quack, 2013). De maneira complementar, Djelic e Sahlin-Andersson (2006a, 2006b) salientam que, nesse tipo de governança, as bases territoriais e a autonomia nacional não podem ser encaradas como óbvias. Suas atividades estão imersas em múltiplas e interativas redes institucionais, embora presentes em estruturas geopolíticas particulares. Logo, é cada vez mais difícil separar o que ocorre dentro dos limites nacionais e o que ocorre através ou além, uma vez que há um emaranhamento de tais limites. Os campos de governança transnacional atravessam as fronteiras nacionais e, por sua vez, interligam atores situados em sociedades distintas que se envolvem em uma variedade de modos de regulação transnacional (Quack, 2013).

À vista disso, a transnacionalização lida com uma configuração em que não há uma hierarquia clara de poderes da definição de regras, mas uma incerteza frequente do que esperar de outros atores. Tampouco se tem estabelecida a legitimidade que tais atores têm ou deveriam ter (Dobusch, Mader, Quack, 2013). O desafio, portanto, consiste em equilibrar o alcance global com a adaptação local (Shenkar, 2004).

De igual modo, a regulação transnacional é apenas um aspecto de todo um campo do fenômeno transnacional (Djelic, Sahlin-Andersson, 2006b; Zajak, 2013). Ela é um modo de governança que estrutura, guia e controla as atividades e interações humanas e sociais além, através e dentro dos territórios nacionais. No entanto, tais regulamentações estão imersas e são suportadas por outros modos de governança. Elas se expandem também com a difusão de lógicas que envolvem desde as esferas econômicas até as sociais (Djelic, Sahlin-Andersson, 2006b), incluindo também, especificamente neste estudo, a da saúde. A dimensão social, de maneira geral, sempre cruzou, conectou ou transformou fronteiras, de modo que os processos sociais são transfronteiriços muito antes da disseminação do sistema de Estado-nação, o qual, por sua vez, também é moldado transnacionalmente (Zajak, 2013).

Consequentemente, a governança é moldada e impacta uma constelação de atores públicos e privados através de diferentes campos transnacionais, que incluem Estados, organizações internacionais, associações profissionais, grupos de especialistas, grupos da 
sociedade civil e corporações empresariais (Djelic, Sahlin-Andersson, 2006b; Dobusch, Mader, Quack, 2013; Quack, 2013). Processos transnacionais podem incluir ainda movimentos sociais, organizações não governamentais (ONGs), migrações, comunidades, cidadania, religiões ou várias práticas culturais, de modo que os vários atores, públicos e privados, dão forma ao significado e à prática dos conceitos de governança (Zajak, 2013).

Os diversos regimes e arranjos transnacionais de governança abrem espaços para que diferentes atores compreendam, usem e adaptem as regras globais às suas circunstâncias locais. Campos transnacionais incluem conjuntos mais amplos de atores (Dobusch, Mader, Quack, 2013). Uma grande variedade de grupos de stakeholders se esforça para moldar as regras transnacionais e sua implementação particular (Quack, 2013). Logo, embora os Estados-nação sejam ativos, eles são apenas um tipo de ator entre vários envolvidos. Eles são incorporados e limitados por atividades e atores reguladores. Agências do Estado negociam com associações sem fins lucrativos, organizações internacionais e atores corporativos, cujas interações são complexas, densas e multidirecionais. A alocação de responsabilidades entre eles está em fluxo, e as fronteiras entre as esferas pública e privada são cada vez mais fluidas (Djelic, Sahlin-Andersson, 2006b).

A análise de campos transnacionais, assim, abrange complexidade, constelações de atores e misturas de modos de governança que não só se afastam da noção de estabilidade como evidenciam grande variação entre os campos, evoluindo ao longo do tempo (Dobusch, Mader, Quack, 2013). A evolução e a expansão da governança transnacional ocorrem por meio de uma espiral de autorreforço. Regulamentação, acompanhamento e avaliação das atividades retroalimentam maiores necessidades, que levam a uma maior regulamentação e governança (Djelic, Sahlin-Andersson, 2006a).

A interdependência e o emaranhamento característicos desses processos refletem, em parte, a rerregulação enquanto a conduzem ainda mais. Consequentemente, maiores interdependência e emaranhamento promovem a necessidade de comparações e padrões de referência sistemáticos, tornando assim necessário aumentar a coordenação entre países e regiões. Isso, por sua vez, gera ainda mais atividades regulatórias (Djelic, Sahlin-Andersson, 2006b). Em razão disso, comparações transnacionais são esclarecedoras porque mostram grande variação em todo o globo no que diz respeito ao surgimento e à transformação de padrões regulatórios (Djelic, Sahlin-Andersson, 2006b). Também são esclarecedores estudos que rastreiam os desenvolvimentos históricos, os processos e as interações discursivas e controversas que conduzem à evolução dos regimes voláteis de governança transnacional (Zajak, 2013).

Quack (2013) salienta que a consideração das trajetórias históricas dos campos de governança transnacionais proporciona compreensão mais situada e diferenciada. Para o autor, quatro dimensões constituem a formação dessas trajetórias: (a) as constelações de atores durante o surgimento do campo; (b) a face do poder de especialização que o projeto pretende e como ele se institucionaliza no quadro teórico organizacional e processual, mediando assim o processo legislativo recursivo; (c) os protestos e conflitos sustentados que geram demanda para o desenvolvimento do campo de governança, muitas vezes decorrentes de crises percebidas; e (d) a capacidade de resposta ou resistência da organização de governança a esses tipos de demandas. 
Portanto, a regulamentação transnacional pode não apenas afetar aqueles que são diretamente regulados, mas outros atores societais e as relações locais de poder. Em função disso, a análise futura deve considerar as consequências involuntárias ou imprevistas dos acordos de governança transnacional e a complexidade da dinâmica regulatória multinível. Há necessidade de mais estudos empíricos, bem como conceituações teóricas que ajudem a tornar esses processos mais compreensíveis em questões, campos e países (Zajak, 2013), ratificando-se com isso a relevância do presente estudo.

\section{Procedimentos metodológicos}

Considerando o objetivo da pesquisa, elaborou-se um resgate histórico do parto normal no Brasil para compreender a influência da transnacionalização ao longo dos anos. Para tal, realizou-se uma pesquisa bibliográfica baseada em dados secundários, de natureza qualitativa-descritiva. Quanto à perspectiva temporal, adotou-se aqui o recorte seccional com avaliação longitudinal, isto é, apesar de o estudo abordar a análise temporal, os dados foram coletados em momentos posteriores ao seu acontecimento e utilizados para explicar a configuração atual do fenômeno (Vieira, 2004).

A coleta de dados abrangeu artigos, livros, dissertações, teses, manuais médicos e relatos das áreas da saúde que auxiliassem na construção histórica. Também foram consultados os sites da ANS, do Ministério da Saúde, do CFM e da Federação Brasileira das Associações de Ginecologia e Obstetrícia (Febrasgo), bem como sites de alguns grupos sociais que se destacam no movimento da humanização. $\mathrm{O}$ foco foi levantar documentos que tratassem do sistema obstétrico brasileiro ao longo dos anos.

Para garantir a cobertura completa deste tipo de pesquisa, também se realizou uma pesquisa manual por edição das últimas cinco décadas em quatro periódicos brasileiros, para descrição do tratamento dado ao assunto parto normal e cesárea no país. Foram pesquisados três periódicos acadêmicos, a Revista de Saúde Pública (com publicações de 1967 até 2015), a revista Femina (1973 a 2011), a Revista Brasileira de Ginecologia e Obstetrícia (1979 a 2015), e uma revista popular, a Veja (1968 a 2015). O critério de escolha dos periódicos foi o período de acervo disponível, dando preferência aos que eram mais abrangentes, por proporcionarem maior aprofundamento longitudinal. Para escolha dos periódicos científicos também se considerou a área de pesquisa, sendo requisito ser da área da saúde, e a nota Qualis atribuída. As informações principais dos artigos foram preenchidas em planilha do Excel, para posterior análise, a qual foi conduzida a partir do quadro teórico aqui abordado.

\section{Sistema obstétrico brasileiro: histórico, mudanças e movimento de humanização}

A análise da história do atendimento ao parto no Brasil demonstra que, até as mudanças do período industrial, as vivências do parto eram de caráter íntimo e privado, sendo uma experiência compartilhada entre mulheres. A parturiente era acompanhada durante toda a gestação e o parto por mulheres mais experientes que, geralmente, já haviam vivenciado o parto. Destacavam-se entre elas também as "aparadeiras", "comadres" ou "parteiras-leigas", detentoras de um saber empírico, tradicional, um conhecimento oral e gestual, transmitido 
de geração em geração. O parto era um evento de espaço doméstico; as parteiras assistiam as mulheres em seus domicílios durante a gestação, o trabalho de parto e os cuidados pósparto, auxiliando também nos cuidados com o recém-nascido (Brenes, 1991; Brasil, 2001).

A obstetrícia ocidental moderna, assim, teve sua origem no conhecimento acumulado pelas parteiras. Os médicos, ou cirurgiões-barbeiros, eram admitidos para ajudar apenas diante de complicações, de modo que a participação do homem foi pouco frequente até o início da era moderna. Foi apenas durante o século XX que a instituição médica consolidou seu controle no processo reprodutivo. O atraso da obstetrícia médica em relação ao desenvolvimento da medicina como um todo sofreu grande influência dos paradigmas da assistência ao parto, pois, por ser interpretado como um processo natural, sua assistência permaneceu, durante longo tempo, desvinculada da prática médica-cirúrgica oficial (RNFS, 2002).

O processo de institucionalização do parto iniciou-se a partir da década de 1940, não se consolidando antes de meados dos anos 1960. Ele teve como principal preocupação a saúde materna restrita à assistência ao parto (Brasil, 2001; Jones, 2007). Segundo Matos et al. (2013), no contexto mundial, esse processo relaciona-se ao fim da Segunda Guerra Mundial, quando os governos da época identificaram a necessidade de reverter as altas taxas de mortalidade materna e infantil. Assim, as iniciativas em busca da redução, aliada às várias conquistas científicas na área da saúde e à introdução da medicina preventiva, produziram um cenário favorável à entrada do saber médico no nascimento humano. A tecnologia substituía o lugar ocupado pela intuição e pela experiência (Brasil, 2001; Jones, 2007), e o parto tornava-se um evento hospitalocêntrico com intensa medicalização e rotinas cirúrgicas (Matos et al., 2013).

Historicamente, o início da incorporação das atribuições obstétricas pela medicina se deu na Europa e, posteriormente, estendeu-se ao Brasil, ao serem inauguradas as escolas de medicina e cirurgia com a chegada da Corte portuguesa ao país (Brenes, 1991). Entretanto, para que a obstetrícia alcançasse legitimidade como ramo da medicina, a gravidez e o parto passaram de um evento fisiológico normal a um procedimento médico, sendo considerados como estados patológicos que requeriam intervenção obstétrica com instrumentos e técnicas cirúrgicas. Logo, foi necessário não somente estabelecer a validade dessas novas técnicas para o parto, mas garantir que sua segurança estava nas mãos do obstetra (RNFS, 2002; OMS, 1996).

Inicialmente, entretanto, a busca por médicos para atendimento de partos era pequena, em decorrência da falta de um cenário para as atividades práticas da obstetrícia e do constrangimento das mulheres em se exporem a outros homens que não os seus cônjuges. Isso fez com que o ensino médico obstétrico permanecesse estagnado por mais de sessenta anos. Tal cenário começou a ser revertido com a popularização do fórceps, que fez com que a participação masculina se tornasse predominante (Dal Molin, 2008). O fórceps foi criado pelos irmãos Chamberlen, na Inglaterra, e posteriormente disseminado por outros países, com a função de auxiliar a saída do bebê pelo canal vaginal. Ele é apontado como o marco inicial da mudança do nascimento, pois sua introdução no parto possibilitou a mudança de uma postura predominantemente expectante para a noção de que era possível controlar o nascimento/natureza, firmando uma nova concepção na consciência popular (RNFS, 
2002; Jones, 2007). Similarmente, outros avanços, como o progresso do conhecimento em fisiopatologia da contração uterina, também ajudaram a fortalecer essa nova mentalidade, ao propiciar o encurtamento e indução do trabalho de parto (Rezende, 1969).

Outrossim, tendo em vista a necessidade da presença das mulheres nas clínicas e maternidades para avanço do conhecimento e prática obstétrica, os médicos buscaram o apoio do governo também quanto aos processos de internação, controle e cadastramento nas clínicas, tendo o controle sobre os nascimentos e incentivando o parto hospitalar em detrimento do domiciliar, principalmente das mulheres mais pobres, que teriam maior facilidade no novo ambiente (Brenes, 1991).

Assim, a prática cada vez mais recorrente das cesáreas ao longo dos anos levou à instituição de uma cultura pró-cesárea entre a população em geral e entre os médicos (Brasil, 2001), reforçando sua predominância. Entre os médicos, alguns dos fatores determinantes que levaram a esse uso excessivo são: maior lucratividade; maior conveniência e previsibilidade; interesses comerciais com pesquisas relacionadas ao uso de tecnologia e maior faturamento com cirurgia; formação do médico obstetra - há cada vez menos partos normais para aprendizado e prática dos obstetras. Já entre as mulheres, as causas apontadas para a maior aceitação por cesárea são: possibilidade de realização conjunta da laqueadura tubária (método contraceptivo permanente); inserção feminina no mercado de trabalho e possibilidade de planejar as licenças no trabalho; e valores culturais, como medo da dor do parto normal, valorização da imagem corporal, fascínio pela tecnologia e conceito popular de que o parto vaginal é mais arriscado para o feto do que uma cesárea (Faúndes, Cecatti; 1991; Rattner, 1996; Castilho, 2000; Brasil, 2001; Jones, 2007).

Vinculado a este último fator, Pereira (2006), Freire et al. (2011) e Leal e Gama (2012) apontam também a falta de informação e o poder hegemônico dos médicos, dado que, apesar de muitas mulheres expressarem o desejo por um parto normal, livre de intervenções, elas geralmente são influenciadas pelo discurso do risco dominante, do medo de sofrerem violência obstétrica e da dor do parto, desconhecendo a fundo as etapas do parto normal, seus riscos e alternativas para lidar com a dor. Portanto, a atuação conjunta desses fatores levou a vigorar o modelo tecnocrático de atenção ao parto e nascimento. Esse modelo deriva de uma visão mecanicista da realidade, gerada a partir da revolução científica, e de dominância nos EUA (Santos, 2002; Rattner, 2009).

No entanto, mesmo com os avanços no conhecimento e a incorporação de novas tecnologias, a mortalidade materna no Brasil tem se mantido constante e muito superior à dos países desenvolvidos nos últimos vinte anos (Rattner, 2009). Vários estudos vinculam taxas maiores de mortalidade materna e neonatal aos altos índices de cesarianas. Faúndes e Cecatti (1991) apresentam pesquisas realizadas em cidades de EUA, França, Suécia e Brasil que relacionaram o risco de morte materna ao tipo de parto. Um resultado em comum foi a maior mortalidade e morbidade materna entre mulheres submetidas à cesárea, com maiores índices no Brasil. Complementarmente, Villar et al. (2006) realizaram uma pesquisa em oito países da América Latina, dentre os quais o Brasil, na qual encontraram associação positiva da cesárea com o tratamento antibiótico pós-parto, a morbidade e a mortalidade materna severa, o aumento de taxas de mortalidade fetal e o aumento do número de bebês internados em cuidado. 
Segundo esses estudos, o uso excessivo da cesárea, principalmente sem necessidade médica evidente, apresenta como desvantagens: maiores riscos de interromper prematuramente a gravidez; síndrome da angústia respiratória devido à imaturidade dos pulmões do bebê; maior incidência de infecções puerperais e de complicações anestésicas, consequentemente, maiores taxas de mortalidade e morbidade materna e neonatal; maior dificuldade de recuperação pós-parto, levando a um período maior de separação entre mãe e bebê, demora no primeiro contato entre eles e no início da amamentação; e consequências econômicas, visto que a cesariana envolve mais profissionais e tem custos mais elevados.

À vista disso, esses riscos e prejuízos têm provocado diversas ações governamentais para mudar o cenário (Castilho, 2000; Jones, 2007). Embora o modelo obstétrico atual ainda seja fortemente orientado pelo atendimento hospitalizado e medicalizado e com alta incidência de cesarianas, cresce nas últimas três décadas um movimento internacional que propõe modificá-lo. Tal movimento tem como base consensual as propostas da OMS, difundidas a partir de 1985 (Silveira, Camargo, Crepaldi, 2010).

As Nações Unidas declararam 1979 como o Ano Internacional da Criança, quando o Comitê Regional para a Europa expressou preocupação quanto a uma série de questões recorrentes - tecnologia em rápida expansão aplicada ao nascimento; duplicação ou mesmo triplicação da taxa de cesariana na década de 1970; demandas crescentes dos grupos de mulheres para retomar o controle sobre suas experiências de parto, entre outras. Como resultado, a OMS decidiu realizar pesquisas sobre serviços perinatais e desenvolver recomendações para a tecnologia apropriada para o nascimento (Wagner, 1991 citado em OMS, 1996). Assim, em 1985, peritos em obstetrícia de todas as regiões da OMS e da Organização Pan-americana de Saúde (Opas) realizaram em Fortaleza (CE) uma conferência, na qual fizeram uma série de recomendações à luz dos conhecimentos da época e da medicina baseada em evidências sobre as práticas relacionadas ao parto normal (OMS, 1996).

A definição desse guia impactou profundamente o Brasil, impulsionando o movimento de humanização do parto. Suas recomendações deram origem a conflitos, manifestações, denúncias e, por conseguinte, novas regulamentações para realinhamento nacional. Nas décadas de 1980 e 1990, iniciou-se uma grande difusão de ações preocupadas com a intensa medicalização do nascimento, reivindicando a preservação da normalidade do parto, menos intervenção e mais proximidade, tudo justificado a partir do guia da OMS. Em 1984, foi instituído o Programa de Assistência Integral à Saúde da Mulher (Paism) (Brasil, 2001). Foi incentivado também o apoio ao parto normal por meio do pagamento da anestesia pelos serviços do Sistema Único de Saúde (SUS) e do atendimento ao parto pela enfermeira obstetriz. Essas profissionais passaram a ter um papel notável no sucedimento dos partos, tanto no acompanhamento e apoio às gestantes durante o trabalho de parto quanto na própria assistência ao parto, resgatando as funções das parteiras de antigamente (Leal, Gama, 2012).

Em 1999, o Ministério da Saúde instituiu o prêmio Galba de Araújo, que reconhece hospitais que se destacaram nas áreas de atendimento humanizado à mulher e ao recémnascido, e de estímulo ao parto normal e ao aleitamento materno (RNFS, 2002). No ano 2000 foi regulamentado, pelo Ministério de Saúde, o Programa Nacional de Humanização 
da Assistência Hospitalar (PNHAH) e feita a inclusão da humanização na pauta da $11^{\mathrm{a}}$ Conferência Nacional de Saúde. Também foi instituído o Programa de Humanização no Pré-natal e Nascimento (PHPN), que prioriza o parto normal, a não medicalização do parto e a redução de intervenções cirúrgicas; incentiva o parto como um processo fisiológico e critica procedimentos que levam à despersonalização da mulher e à realização de cesáreas desnecessárias (Griboski, Guilhem, 2006). Todos esses critérios e medidas de avaliação foram delimitados à luz das recomendações da OMS (1996).

Ainda reflexo desse guia, em 2005 foi promulgada a lei n.11.108, conhecida como Lei do Acompanhante. A partir de pesquisas em várias regiões - a saber, Toronto (Hodnett, Osbom, 1989); Finlândia (Hemminki et al., 1990); África do Sul (Hofmeyr et al., 1991); Guatemala (Klaus et al., 1986) -, que apontaram que o apoio físico e empático contínuo durante o trabalho de parto apresentava muitos benefícios, a OMS passou a recomendar a presença de um acompanhante no parto. Essas evidências deram suporte para a promulgação da lei supracitada, que garante às parturientes o direito à presença de um acompanhante de sua escolha durante o trabalho de parto, o parto e o pós-parto (Brasil, 7 abr. 2005; Rattner, 2009; OMS, 1996; RNFS, 2002).

Com essa lei, o Ministério da Saúde lançou uma portaria no mesmo ano (Brasil, 2 dez. 2005), e a ANS em 2007 (ANS, 9 jan. 2007), autorizando os hospitais e planos de saúde a estenderem a cobertura de acomodação e refeições ao acompanhante. Similarmente, diversos projetos de leis municipais aprovaram a presença de doulas em maternidades municipais e hospitais privados. As doulas são profissionais que oferecem informação, apoio emocional e conforto físico às gestantes, auxiliando durante o pré-natal, o trabalho de parto, o parto e o pós-parto.

Outra medida tomada pelo Ministério da Saúde foi a definição de um teto percentual decrescente para o pagamento de cesáreas aos hospitais. Em 1998, foi editada a portaria n.2.816, que limitou o pagamento de cesarianas a 40\%, passando a 35\% em 1999, e chegando a $30 \%$ no primeiro semestre de 2000 . A iniciativa contribuiu para estabilizar ou reduzir as taxas, ao menos nos serviços do SUS e conveniados, contrariamente ao que ocorria nos serviços privados, em que essas taxas continuavam aumentando (RNFS, 2002; MPF, 2010). Mais recentemente, então, o Ministério da Saúde, junto à ANS, passou a intervir também no âmbito privado.

Em julho de 2015 entrou em vigor a resolução n.368, visando reduzir o número de cesarianas desnecessárias (Brasil, 6 jan. 2015), e em fevereiro de 2016, com o objetivo de atender uma decisão judicial do Tribunal Regional Federal da $3^{\text {a }}$ Região, a ANS promulgou a resolução normativa n.398 (Brasil, 5 fev. 2016). Essa dispõe sobre a obrigatoriedade de credenciamento de enfermeiros obstétricos/obstetrizes por operadoras de planos privados de assistência à saúde e hospitais que constituem suas redes, e reforça a obrigação, prevista na norma anterior, de entrega pelos médicos de documentos e orientações às parturientes, com o objetivo de esclarecer os riscos e benefícios da cesariana e do parto normal (Brasil, 5 fev. 2016).

Adicionalmente, o CFM demonstrou preocupação com o cenário obstétrico brasileiro, passando a intervir também, no ano de 2016, por meio da resolução n.2.144. Essa define que a cesariana a pedido da paciente só pode ser realizada a partir da 39ª semana de gestação 
(CFM, 22 jun. 2016). A adoção do marco de 39 semanas se dá por ser o período em que os estudos analisados pelo Defining "Term" Pregnancy Workgroup, organizado pelo Colégio Americano de Obstetras e Ginecologistas (Acog, na sigla em inglês), demonstram que o bebê já pode estar maduro. A resolução aprovada pelo CFM também consultou como fonte o Instituto Nacional de Saúde da Criança e Desenvolvimento Humano (NICHD, na sigla em inglês) dos EUA, que afirma que, entre 37 e 39 semanas, o bebê atravessa uma fase crítica de desenvolvimento do cérebro, dos pulmões e do fígado (CFM, 20 jun. 2016).

Diversos estudos de periódicos da saúde e medicina também apoiam essas medidas, evidenciando preocupações com a alta incidência de cesáreas e vinculando-as a maiores riscos. No ano de 2012, foi realizada a primeira pesquisa a oferecer um panorama nacional sobre a situação da atenção ao parto e nascimento no Brasil, coordenada pela Fundação Oswaldo Cruz. Os resultados identificados ratificam urgência em reformar o modelo em vigor no país, a partir de uma série de recomendações a gestores e entidades de classe, profissionais da saúde, universidades e instituições de pesquisa, movimentos sociais, famílias e sociedade em geral, pautadas novamente nas diretrizes da OMS e do Ministério da Saúde, com suporte de comparativos com outros países (Leal, Gama, 2012). No entanto, percebe-se que essas manifestações discursivas não têm sido suficientes para reverter tal cenário na prática, o que é ilustrado por meio dos índices crescentes de cesarianas e da maior atuação de movimentos sociais descritos a seguir.

\section{Atuação de movimentos sociais}

O movimento pela humanização do parto caracteriza-se por sua diversidade, com multiplicidade de perspectivas, iniciativas e atores envolvidos (Diniz, 2005). Além das medidas adotadas por órgãos governamentais, tratadas anteriormente, no Brasil há forte participação dos movimentos de mulheres (Diniz, 2005; Tornquist, 2004), assim como ocorre internacionalmente (Goer, 2004), evidenciando-se uma constelação de atores públicos e privados através de diferentes campos transnacionais (Djelic, Sahlin-Andersson, 2006b; Dobusch, Mader, Quack, 2013).

Goer (2004) salienta que em todo o mundo há grupos de ativistas de nascimento e que, apesar das grandes diferenças na cultura e nos sistemas de assistência à maternidade, os propósitos são notavelmente semelhantes. A maioria se formou para reparar as falhas da gestão obstétrica convencional em fornecer atendimentos seguros, efetivos e humanizados e tentar mudar as práticas e políticas que evidências científicas demonstram ferir ou mesmo pôr em risco a vida de mães e bebês.

Dessa forma, mesmo antes das iniciativas governamentais, outros movimentos no Brasil já atuavam na sociedade, reivindicando e defendendo a humanização do parto e do nascimento (Brasil, 2001; Jones, 2007). Em 1993, alguns grupos do movimento brasileiro de mulheres contribuíram para a fundação da Rede pela Humanização do Parto e Nascimento (ReHuNa), a fim de resgatar o nascimento humanizado e as condutas e posturas relativas ao parto, reunindo centenas de participantes, entre indivíduos e instituições (Diniz, 2005; Jones, 2007; RNFS, 2002). A rede foi fundada em Campinas, no sítio de um dos médicos que atuava no hospital da universidade local e que, sendo referência nacional, tinha 
contato com várias pessoas do Brasil que de alguma maneira trabalhavam diferentemente do modelo hegemônico do país (Tornquist, 2004).

O encontro envolvia profissionais da saúde que tratavam de questões relativas à saúde da mulher, à gestação e ao nascimento. Nos últimos dez anos, a ReHuNa tem contribuído para facilitar a mudança de práticas obstétricas por meio de formação, educação, organização de campanhas e orientação na implantação de serviços. A organização de eventos, como o Encontro Parto Natural e Consciente no Rio, contribuiu também para formação de uma comunidade que disseminou a formação de outros grupos, como a organização da rede em Santa Catarina, a Pró-ReHuNa (RNFS, 2002), e que, com a propagação da internet, auxiliam na atuação e divulgação em redes sociais de eventos, artigos, relatos e abaixo-assinados (Goer, 2004; Tornquist, 2004).

Em 2001, um grupo de mulheres de São Paulo - mães e profissionais da saúde (médicas obstetras, psicólogas, doulas) - criou o site eletrônico Amigas do Parto, buscando informar mulheres e profissionais a respeito da assistência ao parto. Similarmente, a rede Parto do Princípio foi formada buscando atuar e defender a promoção dos direitos sexuais e reprodutivos da mulher. Uma das ações desempenhadas foi mapear grupos de apoio presencial à gestante em todo o Brasil que oferecem encontros gratuitos e periódicos e que reúnem gestantes, casais, pais e mães recentes, além de profissionais de saúde convidados, formando a Rede Gapp (Grupo Apoiado pela Parto do Princípio). Atualmente, a Rede Gapp tem mais de quarenta grupos presenciais, atendendo mais de trinta cidades brasileiras.

Esses grupos são formados em sua maioria por mães e profissionais da saúde (médicas, enfermeiras, psicólogas, doulas). Eles tomam como base as evidências científicas, diretrizes e parâmetros médicos determinados principalmente pela OMS (Griboski, Guilhem, 2006; RNFS, 2002), e em seus veículos de divulgação (sites, redes sociais) há grande disseminação de artigos e pesquisas internacionais. Muitos desses arquivos são de autoria de profissionais da saúde membros dos próprios grupos ou de outros que se destacam no movimento em prol da humanização, tanto nacional quanto internacionalmente. Vários desses atores possuem vínculos transnacionais, por meio de redes, formação profissional, participação em eventos, congressos e pesquisas, que dão base para gerar condições ideais, organizacionais e institucionais para a mobilização em contextos desfavoráveis (Vieira, Quack, 2016).

Ademais, o movimento das mulheres tem forte relação com ações feministas no Brasil, a exemplo da Rede Feminista de Saúde, ativa desde o início dos anos 1990. A inserção de feministas em associações e sindicatos favoreceu o estabelecimento de alianças com outros movimentos e de parcerias entre ONGs e órgãos governamentais, abrindo canais de participação e ampliação de políticas públicas. Tais parcerias têm contribuído por meio do envolvimento na implementação de políticas públicas nas áreas da saúde e da justiça, bem como de denúncias quanto às políticas e práticas de saúde do país (Tornquist, 2004; Jones, 2007).

O Paism foi instituído em resposta à demanda do movimento organizado de mulheres. Do mesmo modo, a abertura da Maternidade Leila Diniz, no Rio de Janeiro, também foi fruto da iniciativa de um grupo composto por várias pessoas ligadas à ReHuNa (Tornquist, 2004). Adicionalmente, as evidências que levaram a OMS a recomendar a presença de acompanhante inspiraram a ReHuNa a iniciar uma campanha em 2000 por esse direito, a 
qual contou com o apoio da Rede Nacional Feminista de Saúde Direitos Sexuais e Direitos Reprodutivos, da Associação Brasileira de Obstetrizes e Enfermeiras Obstetras e da União dos Movimentos Populares de Saúde de São Paulo. A campanha foi se ampliando em âmbito nacional, e membros da ReHuNa, juntamente com seus aliados, passaram a apresentar projetos de lei em seus estados (RNFS, 2002), influenciando, em 2005, na criação da lei n.11.108/2005, supracitada.

Outro exemplo dessa influência corresponde a denúncias prestadas pela Rede Parto do Princípio junto ao Ministério Público Federal (MPF). Em 2006, essa rede elaborou e entregou ao MPF um dossiê sobre o atendimento ao parto no Brasil, citando mais de trinta referências a artigos científicos e solicitando a atuação do ministério diante do cenário de abuso de cesáreas. No ano seguinte, ativistas da rede participaram de uma audiência pública em São Paulo promovida pelo MPF a fim de debater o assunto com representantes de Ministério da Saúde, ANS, CFM, Associação dos Hospitais Privados, Federação Brasileira de Hospitais, Conselho Federal de Enfermagem, Escola Paulista de Medicina e de planos de saúde (Denúncia..., s.d.). Com base no dossiê e na audiência, o MPF emitiu uma ação pública em 2010, cobrando da ANS a regulamentação dos planos de saúde, com destaque para o credenciamento de enfermeiras obstetras, sua inserção na assistência ao parto, o incentivo de práticas humanizadas no parto e nascimento e o pagamento diferenciado entre parto normal e cesárea (MPF, 2010).

Além de sua atuação nacional, é notável também que os movimentos sociais tenham atravessado fronteiras, interligando atores situados em sociedades distintas (Quack, 2013), com impacto internacional. No final do ano 2000, a Rede ReHuNa organizou, juntamente com outros atores institucionais, o congresso de Fortaleza, intitulado Conferência Internacional sobre Nascimento e Parto. A partir daí, o movimento pela humanização ganhou amplitude internacional com a criação da Rede Latino-americana e Caribenha para a Humanização do Parto e do Nascimento (Relacahupan) durante o evento. Também foi organizado pela ReHuNa um acordo com a Agência Jica de Cooperação Internacional (Japan International Cooperation Agency) (Tornquist, 2004). Esta forneceu apoio em diversas iniciativas governamentais, como a criação do Paism, a internacionalização do Hospital Sofia Feldman e a realização do Curso Internacional de Assistência Humanizada à Mulher e ao Recém-nascido, que visou ao melhoramento e à criação do modelo de assistência humanizada ao parto e nascimento na América Latina e nos Países Africanos de Língua Oficial Portuguesa (Mourão, 2010).

\section{Discutindo a transnacionalização do parto normal}

Este estudo contribui com a perspectiva da transnacionalização ao demonstrar que as regulamentações e práticas inseridas na fronteira nacional recebem influências que atravessam tais fronteiras. Contextos internacionais influenciam a forma como regulamentações nacionais são aplicadas em um território, demonstrando a inter-relação entre dimensões política, econômica, legal e social, permeadas pela transfronteirização. Por meio do histórico do sistema obstétrico brasileiro, pôde-se visualizar o impacto do cenário internacional nas diretrizes e práticas adotadas no país. Por mais que o cenário obstétrico brasileiro tenha 
evidenciado claramente um sistema dominante (Rattner, 2009) - uma vez que ele se distanciava de padrões internacionais -, desde a década de 1980, e mais fortemente a partir dos anos 2000, iniciativas governamentais e sociais passaram a vigorar sustentadas por estudos realizados em outros países (Hemminki et al., 1990; Faúndes, Cecatti, 1991; Hofmeyr et al., 1991; Villar et al., 2006; Leal, Gama, 2012).

A definição de novas normas e regulamentos ao longo dos anos (MPF, 2010; Brasil, 2015; Brasil, 5 fev. 2016; CFM, 22 jun. 2016) permite uma melhor compreensão de como as regras e instituições evoluem além e através das fronteiras (Dobusch, Mader, Quack, 2013). Tanto os documentos oficiais emitidos por órgãos governamentais quanto aqueles elaborados e divulgados por movimentos sociais (Denúncia..., s.d.) foram construídos e embasados, prioritariamente, com base em comparações com índices divulgados pela OMS e com outros países, ilustrando o engajamento local, mas com esforços de governança que avançam as fronteiras (Dobusch, Mader, Quack, 2013), como pode ser visualizado nos trechos a seguir:

Em primeiro lugar, não há como negar que 'as taxas de cirurgia cesariana praticadas no setor privado de saúde estão muito acima do recomendado pela OMS e não encontram similar em qualquer outro lugar do mundo' (MPF, 2010, p.3; destaque nosso).

O Conselho Federal de Medicina juntou aos autos da Representação n.1.34.001.004458/ 2006-98, fls. 380, artigo de renomado obstetra a respeito do espantoso índice de cesárea brasileiro, destacando-se o seguinte trecho: 'O que nos assusta é constatar que, em centros obstétricos de alto risco, em países de primeiro mundo, onde as implicações legais de complicações neonatais e obstétricas são onerosas para o médico, a incidência da via abdominal para o parto situa-se em torno de $15 \%$, enquanto que em nosso meio, mesmo em gestações de baixo risco, atingem-se cifras próximas de 100\%' (MPF, 2010, p.3; destaques do original).

Os trechos transcritos auxiliam a compreender como a evolução da regulamentação em países individuais, no caso o Brasil, estende-se a comparações transnacionais. Ao serem confrontados os cenários local e global, é possível identificar emergência e transformação de padrões regulatórios (Djelic, Sahlin-Andersson, 2006b) nacionais para irem ao encontro dos internacionais. O campo transnacional em relação ao parto normal se caracteriza por grande variação e evolução ao longo do tempo, que, impactadas por cenários de outros países, acabou por gerar modelos transnacionais e quadros institucionais (Djelic, SahlinAndersson, 2006b) materializados, por exemplo, no guia da OMS de 1996.

Os índices e recomendações divulgados pela OMS, delimitados a partir da revisão de literatura de estudos publicados e realizados em diversos países e regiões (OMS, 1996), evidenciam como a governança transnacional quanto ao parto normal interconectou diversos atores, de múltiplas esferas - da saúde, societal, da política, judicial -, formando um sistema de regras e regulamentações que governa essas relações e que foi responsável por dar forma ao significado e à prática dos conceitos de governança (Zajak, 2013).

Além disso, o fato de tais ações estarem embasadas em pesquisas e evidências científicas e em comparações entre índices de vários países elucida aspectos centrais da transnacionalização: a especialização e a ciência, as quais extrapolam fronteiras físicas. Novos modos de governança são altamente baseados na expertise, sendo legitimados pela 
referência à ciência e expressados em termos de medidas (Quack, 2013), no caso, as altas taxas de mortalidade materna e neonatal, que inicialmente legitimaram a introdução do parto hospitalar e da operação cesariana e, posteriormente, a alta incidência desta e a sua relação com as ainda elevadas taxas de mortalidade. Logo, à medida que tais comparações se demonstraram altamente distantes do contexto internacional, criou-se abertura para protestos e conflitos, os quais geraram demanda para o desenvolvimento de novas regulamentações decorrentes das crises percebidas, defendendo-se, assim, o retorno à normalidade do parto, atendimento humanizado e medicina baseada em evidências.

Inicialmente, foi identificado maior impacto nas redes públicas, com medidas tomadas para hospitais conveniados pelo SUS. No entanto, a trajetória do parto normal no Brasil extrapolou a dimensão governamental/pública. Diante da fluidez entre as fronteiras das esferas pública e privada (Djelic, Sahlin-Andersson, 2006b) e da incidência concomitante de resistência (Quack, 2013), expressa, principalmente, pelos índices alarmantes de cesáreas sem indicação obstétrica no sistema privado (Leal, Gama, 2012), a alocação de responsabilidades para o cenário destoante se deslocou, sequencialmente, para o mesmo, envolvendo profissionais e planos de saúde. A ANS e o CFM interviram com normas e regulamentos que visavam disseminar esses modelos transnacionais também nessas organizações.

Ademais, tais modelos propiciaram a abertura do campo para maior participação do público (Dobusch, Mader, Quack, 2013; Zajak, 2013). Além da forte atuação do Estado, como órgão regulador, a trajetória do parto normal demonstrou a dimensão social influenciando nos conceitos de governança e também cruzando, conectando e transformando fronteiras (Zajak, 2013). Os processos transnacionais passaram a incluir igualmente movimentos sociais, ONGs e redes feministas, com atuação tanto local e nacional como internacional (Relacahupan, Jica, congressos organizados). A partir da identificação com outras culturas e práticas e diante da emergência de situações críticas na área da saúde (Djelic, SahlinAndersson, 2006b), grupos sociais, organizados principalmente por mulheres, demandaram maior regulamentação e monitoramento com base em práticas humanizadas (OMS, 1996).

Atores operando transnacionalmente têm geralmente múltiplas afiliações, inclusive locais e globais. Essa combinação de afiliações trouxe grandes impactos, uma vez que a multiplicidade de imersão permitiu o fluxo de ideias, recursos e estratégias em divisões nacionais, gerando criatividade e novidade (Vieira, Quack, 2016) e suportando suas ações. Assim, a atuação desses grupos e redes demonstrou a capacidade de lutas sociais e políticas em influenciar a direção e os resultados da construção de instituições transnacionais. Foi a partir da forte atuação desses movimentos que muitas iniciativas governamentais foram efetivadas, estreitando as fronteiras transnacionais.

Por conseguinte, conforme sintetizado no Quadro 1, as dimensões que constituem a trajetória (Quack, 2013) do parto normal no Brasil permitem delimitar a expansão deste campo transnacional por meio de uma espiral complexa de autorreforço, marcada por interdependência e emaranhamento (Dobusch, Mader, Quack, 2013; Djelic, SahlinAndersson, 2006a, 2006b). Isto é, a constante comparação a padrões de referência sistemáticos - guia da OMS, índices e pesquisa de outros países -, caracterizados pela interdependência entre o local e o global, levou a uma necessidade crescente de aumentar 
a coordenação entre países por meio de atividades regulatórias, projetos e regulamentos, inicialmente públicos. A delimitação dessas atividades, por sua vez, levou a um maior acompanhamento e avaliação delas, principalmente por movimentos sociais e ONGs, que, diante da constatação de resistência e desalinhamento reincidente (índices crescentes de cesárea), retroalimentaram maiores necessidades, levando a uma maior regulamentação/ re-regulamentação e governança, e envolvendo uma constelação maior de atores (públicos e privados).

Quadro 1: Dimensões da trajetória histórica do parto normal

\begin{tabular}{|l|l|}
\hline Constelações de atores & $\begin{array}{l}\text { Ministério da Saúde, MPF, CFM, ANS, movimentos sociais/ ONGs, mulheres, } \\
\text { profissionais da saúde }\end{array}$ \\
\hline $\begin{array}{l}\text { Face do poder de } \\
\text { especialização }\end{array}$ & $\begin{array}{l}\text { Especialização pautada na medicina baseada em evidências, guia da OMS (1996), e } \\
\text { comparativos com pesquisas e índices internacionais }\end{array}$ \\
\hline $\begin{array}{l}\text { Protestos e conflitos } \\
\text { sustentados }\end{array}$ & $\begin{array}{l}\text { Divergências entre as evidências e índices de padrões internacionais e nacionais, } \\
\text { demanda para mudanças e alinhamento global }\end{array}$ \\
\hline \multirow{5}{*}{$\begin{array}{l}\text { Capacidade de resposta } \\
\text { ou resistência }\end{array}$} & $\begin{array}{l}\text { Respostas: Paism; prêmio Galba de Araújo; PNHAH; PHPN; pagamento da anestesia } \\
\text { e teto percentual para o pagamento de cesáreas pelo SUS; lei n.11.108; resoluções } \\
\text { n.368, 398, 2.144; criação e divulgação de eventos, artigos, abaixo-assinados; } \\
\text { mapeamento de grupos de apoio presencial à gestante; denúncias junto ao } \\
\text { Ministério Público Federal; Curso Internacional de Assistência Humanizada à Mulher } \\
\text { e ao Recém-nascido }\end{array}$ \\
\cline { 2 - 2 } & $\begin{array}{l}\text { Incidência concomitante de resistência, principalmente do setor privado, que ainda } \\
\text { apresenta altos índices de cesariana sem indicação obstétrica adequada }\end{array}$ \\
\hline
\end{tabular}

Fonte: Elaborado pelos autores, com base em Quack (2013).

Portanto, a análise histórica do parto normal possibilita verificar que suas trajetórias e evolução não foram inseridas em um contexto restritivamente nacional, mas sofreram influência de órgãos mundiais, cenários de outros países e pesquisas internacionais, não podendo, dessa forma, serem contempladas isoladamente.

\section{Considerações finais}

Com base na perspectiva da transnacionalização (Djelic, Sahlin-Andersson, 2006a, 2006b; Dobusch, Mader, Quack, 2013) e a partir da análise histórica do sistema obstétrico do Brasil, este estudo retratou a influência da transnacionalização no parto normal no país, focando no impacto que esse tipo de governança teve sobre políticas e regulamentações nacionais, bem como na extensão para a formação e atuação de movimentos sociais. Como resultado, evidenciou-se que a trajetória histórica do parto normal no Brasil apresentou-se a partir da influência e justificativa de estudos e pesquisas internacionais, recomendações de órgãos mundiais e contextos distintos de outros países e regiões. Assim sendo, os limites de atividades locais não se restringiram necessariamente às fronteiras nacionais, já que padrões e recomendações transnacionais passaram a impactar localmente, direcionando a criação de novas regulamentações.

Diante de divergências de cenários - o local e o global - percebidos como crise, criouse uma demanda tanto pública, direcionada pelos órgãos governamentais, quanto social, 
representada por ONGs, redes e grupos de mulheres, que direcionaram e impactaram a resposta do Estado, propiciando a emergência de normativas que visavam alinhar o contexto nacional ao transnacional.

Assim, este estudo contribui ao mostrar a relação dinâmica e autorreforçante entre diversos contextos na geração de regulamentações decorrentes de modelos transnacionais. O contexto do sistema obstétrico no Brasil, com as mudanças ao longo da história e, atualmente, com a dominância de um modelo tecnicista e intervencionista, frente ao crescente movimento de humanização do parto e do nascimento, propiciou a emergência de iniciativas transnacionais sociais e governamentais. Isso evidenciou que, de acordo com Djelic e Sahlin-Andersson (2006a, 2006b), a autonomia nacional não pode ser tida como óbvia, uma vez que suas atividades encontram-se imersas em redes institucionais múltiplas e interativas, mesmo estando inseridas em regiões geopolíticas particulares.

Em função disso, pesquisas em transnacionalização se destacam por sua grande relevância, evidenciando a necessidade de um olhar mais abrangente, já que, como há uma inter-relação de limites, é cada vez mais difícil separar o que ocorre dentro dos limites nacionais e o que ocorre através ou além deles.

Portanto, acredita-se que este estudo contribui para ampliar a abordagem da transnacionalização, ao endereçar a complexidade envolvida em aspectos nacionais que não podem ser considerados isentos de uma inserção ampla em contextos internacionais. As comparações históricas e entre nações revelam as várias formas como as sociedades e culturas encontram-se interconectadas entre fronteiras, expondo suas interdependências. Ao tratar de tais interdependências, este texto permitiu compreender a influência da transnacionalização no parto normal no Brasil, que origina e direciona ações de mudança e instituições de normas e regulamentações de acordo com governança transnacional que atravessam as fronteiras nacionais.

Resta, como possibilidade de estudo, a análise dessa influência em outros países que, apesar de não possuírem índices tão elevados quanto o Brasil, também vivenciam contextos similares de iniciativas em prol da humanização e de lutas sociais com assuntos conectados à maternidade, como é o caso de outras nações da América Latina, com forte atuação da rede Relacahupan (Jones, 2007; Tornquist, 2004), por exemplo. Sugere-se ainda a pesquisa em outros campos empíricos, para investigação das conclusões tratadas aqui.

\section{NOTAS}

${ }^{1}$ A expressão "sistema obstétrico" empregado neste artigo aplica-se ao sistema de atenção médica às mulheres durante o pré-natal, gravidez, parto e pós-parto, incluindo ações discursivas e práticas na relação entre profissionais da saúde e paciente (mulheres) nesses períodos.

${ }^{2}$ Reconhecemos que "parto normal" é uma expressão polissêmica que sofre influência de diversas matrizes político-ideológicas (Dutra, 2005); no entanto, o objetivo do artigo não foi adentrar nessas discussões, e ele é usado aqui para se referir a todo nascimento que ocorra por via vaginal, a partir de um desencadeamento fisiológico.

${ }^{3}$ Nesta e nas demais citações de textos em outros idiomas, a tradução é livre. 


\section{REFERÊNCIAS}

ANS.

Agência Nacional de Saúde Suplementar.

Resolução Normativa n.167, de 9 de janeiro de 2007. Disponível em: <http://www.ans.gov.br>. Acesso em: 2 dez. 2016.9 jan. 2007.

ANS PUBLICA...

ANS publica resolução para estimular parto normal na saúde suplementar. Agência Nacional de Saúde Suplementar, Rio de Janeiro. Disponível em: <http://www.ans.gov.br/aans/noticias-ans/ consumidor/2718-ministerio-da-saude-e-anspublicam-resolucao-para-estimular-partonormal-na-saude-suplementar>. Acesso em: 2 dez. 2016. s.d.

ATUALIZAÇÃO...

Atualização das taxas partos na saúde suplementar. Agência Nacional de Saúde Suplementar. Disponível em: <http://www.ans. gov.br>. Acesso em: 2 dez. 2016. 17 maio 2015.

BARROS, Aluísio J.D. et al.

Patterns of deliveries in a Brazilian birth cohort: almost universal cesarean sections for the better-off. Revista de Saúde Pública, v.45, n.4, p.635-643. 2011.

BETRAN, Ana Pilar et al.

What is the optimal rate of caesarean section at population level? A systematic review of ecologic studies. Reproductive Health, v.12, n.57, p.1-10. 2015.

BRASIL.

Presidência da República. Resolução normativa n.398, de 5 de fevereiro de 2016. Brasília.

Disponível em: <http://www.ans.gov.br>. Acesso em: 9 dez. 2016.5 fev. 2016.

BRASIL.

Presidência da República. Resolução normativa n.368, de 6 de janeiro de 2015. Brasília.

Disponível em: <http://www.ans.gov.br>. Acesso em: 9 dez. 2016.6 jan. 2015.

BRASIL.

Presidência da República. Portaria MS/GM n.2.418, de 2 de dezembro de 2005. Brasília. Disponível em: <http://www.saude.mppr. mp.br>. Acesso em: 2 dez. 2016. 2 dez. 2005.

BRASIL.

Presidência da República. Lei n.11.108, de 7 de abril de 2005. Brasília. Disponível em: <http:// www.planalto.gov.br>. Acesso em: 2 dez. 2016. 7 abr. 2005.

BRASIL.

Ministério da Saúde. Parto, aborto e puerpério: assistência humanizada à mulher. Brasília: Ministério da Saúde. 2001.
BRENES, Anayansi Correa.

História da parturição no Brasil, século XIX. Cadernos de Saúde Pública, v.7, n.2, p.135-149. 1991.

CASTILHO, Sueli A.

O resgate do parto normal: as contribuições de uma tecnologia apropriada. Dissertação (Mestrado em Enfermagem) - Universidade Federal de Santa Catarina, Florianópolis. 2000.

CFM.

Conselho Federal de Medicina. Resolução n. 2.144, de 17 de março de 2016. Diário Oficial da União, n.118, seção 1, p.138. Disponível em: <http://pesquisa.in.gov.br/imprensa/jsp/ visualiza/index.jsp?jornal $=1 \&$ data $=22 / 06 / 2016$ \&pagina $=138>$. Acesso em: 12 nov. 2018. 22 jun. 2016.

CFM.

Conselho Federal de Medicina. CFM define critérios para realização de parto cesariano. Disponível em: <http://portal.cfm.org.br>. Acesso em: 2 dez. 2016. 20 jun. 2016.

DAL MOLIN, Rossano Sartori.

O exercício da atividade da enfermeira obstétrica: análise do discurso médico no Rio Grande do Sul no ano de 2005. Dissertação (Mestrado em Enfermagem) - Universidade Federal do Rio Grande do Sul, Porto Alegre. 2008.

DENÚNCIA...

Denúncia da Parto do Princípio motiva Ação do Ministério Público Federal. Parto do Princípio.

Disponível em: <http://www.partodoprincipio. com.br/den-ncia--altas-taxas-de-ces-reas $>$.

Acesso em: 3 dez. 2016. s.d.

DINIZ, Carmen Simone Grilo.

Humanização da assistência ao parto no Brasil: os muitos sentidos de um movimento. Ciência e Saúde Coletiva, v.10, n.3, p.627-637. 2005.

DJELIC, Marie-Laure; SAHLIN-ANDERSSON, Kerstin.

Institutional dynamics in a re-ordering world. In: Djelic, Marie-Laure; Sahlin-Andersson, Kerstin (Ed.). Transnational governance: institutional dynamics of regulation. New York: Cambridge University Press. p.375-397. 2006a.

DJELIC, Marie-Laure; SAHLIN-ANDERSSON, Kerstin.

Introduction: a world of governance: the rise of transnational regulation. In: Djelic, Marie-Laure; Sahlin-Andersson, Kerstin (Ed.). Transnational governance: institutional dynamics of regulation. New York: Cambridge University Press. p.1-47. 2006b. 
DOBUSCH, Leonhard; MADER, Philip; QUACK, Sigrid.

Introduction. In: Dobusch, Leonhard; Mader, Philip; Quack, Sigrid (Ed.). Governance across borders: transnational fields and transversal themes. Berlin: Epubli. p.17-22. 2013.

DUTRA, Ivete Lourdes.

Parto natural, normal e humanizado: a polissemia dos termos e seus efeitos sobre a atenção ao parto. Dissertação (Mestrado em Enfermagem) - Universidade Federal do Rio Grande do Sul, Porto Alegre. 2005.

FAÚNDES, Aníbal; CECATTI, José Guilherme. A operação cesárea no Brasil: incidência, tendências, causas, consequências e propostas de ação. Cadernos de Saúde Pública, v.7, n.2, p.150173. 1991.

FRASÃO, Gustavo.

Ministério lança protocolo com diretrizes para parto cesariana. Portal da Saúde, Ministério da Saúde. Disponível em: <http://portalsaude. saude.gov.br/index.php/o-ministerio/principal/ secretarias/sas/saude-da-mulher/noticias-saudeda-mulher/23000-ministerio-lanca-protocolocom-diretrizes-para-parto-cesariana $>$. Acesso em: 2 dez. 2016. 2016.

FREIRE, Nara C. et al.

Parto normal ou cesárea? A decisão na voz das mulheres. Revista Baiana de Enfermagem, v.25, n.3, p.237-247. 2011.

GOER, Henci.

Humanizing birth: a global grassroots movement. Birth, v.31, n.4, p.308-314. 2004.

GRIBOSKI, Rejane Antonello; GUILHEM, Dirce. Mulheres e profissionais de saúde: o imaginário cultural na humanização ao parto e nascimento. Texto e Contexto Enfermagem, v.15, n.1, p.107-114. 2006.

HEMMINKI, Elina et al.

A trial on continuous human support during labor: feasibility, interventions and mothers' satisfaction. Journal of Psychosomatic Obstetrics and Gynecology, v.11, n.4, p.239-250. 1990.

HODNETT, Ellen D.; OSBORN, Richard W. A randomized trial of the effects of monitrice support during labor: mothers' views two to four weeks postpartum. Birth, v.16, n.4, p.177183. 1989.

HOFMEYR, G. Justus et al.

Companionship to modify the clinical birth environment: effects on progress and perceptions of labour, and breastfeeding. British Journal of Obstetrics and Gynaecology, v.98, n.8, p.756-764. 1991.
JONES, Ricardo Herbert.

Curso de introdução à humanização do nascimento. [s.l.]: [s.n.]. 2007.

JUNQUEIRA, Eduardo.

Mãe natureza. Veja, n.1.495, p.9-13. Disponível em: <http://veja.abril.com.br/acervodigital/ home.aspx>. Acesso em: 10 set. 2015. 1997.

KLAUS, Marshall H. et al.

Effects of social support during parturition on maternal and infant morbidity. British Medical Journal, v.293, n.6.547, p.585-587. 1986.

LEAL, Maria do Carmo; GAMA, Silvana G.N. Nascer no Brasil: inquérito nacional sobre parto e nascimento. Rio de Janeiro: Ensp/Fiocruz. Disponível em: <http://www6.ensp.fiocruz.br/ nascerbrasil>. Acesso em: 2 dez. 2016. 2012.

MATOS, Greice C. et al.

A trajetória histórica das políticas de atenção ao parto no Brasil: uma revisão integrativa. Revista Enfermagem UFPE, v.7, p.870-878. 2013.

MENDOZA-SASSI, Raúl Andrés et al. Risk factors for cesarean section by category of health service. Revista de Saúde Pública, v.44, n.1, p.80-89. 2010.

MOURÃO, Jusimeire.

Jica, ABC e Hospital Sofia Feldman capacitam profissionais de países da América Latina, Caribe e Países Africanos de Língua Portuguesa em assistência maternoinfantil. Jica Brasil.

Disponível em: <https://www.jica.go.jp/brazil/ portuguese/office/news/2010/21102010.html>. Acesso em: 11 dez. 2016. 2010.

MPF.

Ministério Público Federal. Ação Civil Pública n.0017488-30.2010.4.03.6100, 24 ${ }^{\text {a }}$ Vara Federal Cível de São Paulo. Disponível em: <http://prsp.jusbrasil.com.br/noticias>. Acesso em: 2 dez. 2016. 2010.

\section{NASCER...}

Nascer: a volta da natureza. Veja, n.423, p.8894. Disponível em: <http://veja.abril.com.br/ acervodigital/home.aspx>. Acesso em: 9 set. 2015. 1976.

OMS.

Organização Mundial da Saúde. Assistência ao parto normal: um guia prático. Brasília: Ministério da Saúde. 1996.

PEREIRA, Adriana L.F. Atuação da enfermeira obstétrica na política pública de humanização ao parto no Rio de Janeiro. Revista Mineira de Enfermagem, v.10, n.3, p.233-239. 2006. 
QUACK, Sigrid.

Regime complexity and expertise in transnational governance: strategizing in the face of regulatory uncertainty. Oñati Socio-Legal Series, v.3, n.4, p.647-678. 2013.

RATTNER, Daphne.

Humanização na atenção a nascimentos e partos: breve referencial teórico. Interface: Comunicação, Saúde, Educação, v.13, p.595-602. 2009.

RATTNER, Daphne.

Sobre a hipótese de estabilização das taxas de cesárea do Estado de São Paulo, Brasil. Revista de Saúde Pública, v.30, n.1, p.19-33. 1996.

REZENDE, Jorge de.

Obstetrícia: conceito, propósitos, súmula histórica. In: Rezende, Jorge de. Obstetrícia. Rio de Janeiro: Guanabara Koogan. p.1-20. 1969.

RNFS.

Rede Nacional Feminista de Saúde, Direitos Sexuais e Direitos Reprodutivos. Dossiê humanização do parto. São Paulo: [s.n.]. 2002.

SAKAE, Thiago Mamôru; FREITAS, Paulo Fontoura; D'ORSI, Eleonora.

Fatores associados a taxas de cesárea em hospital universitário. Revista de Saúde Pública, v.43, n.3, p.472-480. 2009.

SANTOS, Marcos Leite dos.

Humanização da assistência ao parto e nascimento: um modelo teórico. Dissertação (Mestrado em Saúde Pública) - Universidade Federal de Santa Catarina, Florianópolis. 2002.

SHENKAR, Oded.

One more time: international business in a global economy. Journal of International Business Studies, v.35, n.2, p.161-171. 2004.
SILVEIRA, Sandra C. da; CAMARGO, Brígido

Vizeu; CREPALDI, Maria Aparecida.

Assistência ao parto na maternidade:

representações sociais de mulheres assistidas e profissionais de saúde. Psicologia: Reflexão e Crítica, v.23, n.1, p.1-10. 2010.

TORNQUIST, Carmen Susana.

Parto e poder: o movimento pela humanização do parto no Brasil. Tese (Doutorado em Antropologia Social) - Universidade Federal de Santa Catarina, Florianópolis. 2004.

VIEIRA, Ana C.A.; QUACK, Sigrid.

Trajectories of transnational mobilization for indigenous rights in Brazil. Revista de Administração de Empresas, v.56, n.4, p.380-394. 2016.

VIEIRA, Marcelo M.F.

Por uma boa pesquisa (qualitativa) em administração. In: Vieira, Marcelo M.F.; Zouain, Deborah M. (Org.). Pesquisa qualitativa em administração. Rio de Janeiro: FGV Editora. p.1328. 2004.

VILLAR, José et al.

Caesarean delivery rates and pregnancy outcomes: the 2005 WHO global survey on maternal and perinatal health in Latin America. The Lancet, v.367, p.1.819-1.829. 2006.

WHO.

World Health Organization. WHO statement on caesarean section rates. Genebra: WHO. 2015.

ZAJAK, Sabrina.

Transnational studies and governance. In: Dobusch, Leonhard; Mader, Philip; Quack, Sigrid (Ed.). Governance across borders: transnational fields and transversal themes. Berlin: Epubli. 2013.

\section{$\rightarrow \rightarrow \rightarrow<<$}

